\title{
An analytical framework for understanding regulatory novelty accompanying allopolyploidization
}

\author{
Guanjing $\mathrm{Hu}^{1}$ and Jonathan F. Wendel ${ }^{1 *}$ \\ ${ }^{1}$ Department of Ecology, Evolution, and Organismal Biology, Iowa State University, Ames, IA \\ 50011 \\ * Author for Correspondence: Jonathan F. Wendel, Department of Ecology, Evolution, and \\ Organismal Biology, Iowa State University, Ames, IA 50011, USA, Tel: 515-294-7172, Fax: \\ 515-294-1337, E-mail address: jfw@,iastate.edu
}




\begin{abstract}
Allopolyploidy is a prevalent process in plants, having important physiological, ecological, and evolutionary consequences. Massive, genome-wide transcriptomic rewiring in response to genomic merger and doubling has been demonstrated in many allopolyploid systems, encompassing a diversity of phenomena, including homoeolog expression bias, genome dominance, expression-level dominance, and revamping of co-expression networks. Here we present an analytical framework to reconcile these patterns of regulatory novelty as governed by distinct sets of intra- and inter-subgenome cis-trans relationships. This approach is a novel extension of classic allele-specific expression analysis to incorporate and distinguish the separate effects of parental regulatory interactions as well as further complications at the allopolyploid level. We demonstrated that the cis-trans framework devised not only offers new perspective on disentangling genetic from epigenetic and higher-order effects that impact gene expression, but also provides the conceptual basis and tools to unify recently presented models for both genomewide expression dominance and biased fractionation in allopolyploids.
\end{abstract}

Keywords: allopolyploidy, allele-specific expression (ASE), cis and trans, homoeolog expression bias, non-additive expression, expression-level dominance 
Polyploidy, or whole-genome duplication (WGD), is exceptionally common in plants, having important physiological, ecological and evolutionary consequences (Stebbins 1940; Levin 1983; Ramsey and Schemske 2002; Leitch and Leitch 2008; Van de Peer et al. 2009; Madlung 2013; Soltis et al. 2014; Van de Peer et al. 2017; Soltis and Soltis 2016). Two types of polyploidy have long been recognized, autopolyploidy, resulting from the multiplication of one progenitor chromosome set, and allopolyploidy, involving hybridization and duplication of divergent parental genomes, classically from different species (Wendel and Doyle 2005). Allopolyploidy in particular is thought to provide avenues for regulatory novelty and hence phenotypic innovation, as evidenced by myriad non-additive and non-Mendelian responses, including gene loss and silencing (Schnable et al. 2011; Freeling et al. 2012; Liu et al. 2014; Mirzaghaderi and Mason 2017; Koh et al. 2010; Szadkowski et al. 2010; Tate et al. 2009; Anssour et al. 2009; Buggs et al. 2009; Eilam et al. 2009); activation of transposable elements (Senerchia et al. 2015; Parisod et al. 2010; Kawakami et al. 2010); epigenetic modifications (Song et al. 2017; Jackson 2017; Madlung et al. 2002; Chen 2007; Salmon et al. 2005; Rapp and Wendel 2005; Bottley 2014; Fulnecek et al. 2009; Kovarik et al. 2008; Yu et al. 2010; Shcherban et al. 2008; Wang et al. 2017; Zhao et al. 2011); and massive, genome-wide transcriptomic rewiring. The latter encompasses a diversity of phenomena, including biased expression of homoeologs on a genic (Yoo and Wendel 2014; Flagel et al. 2008; Akama et al. 2014; Combes et al. 2013; Wang et al. 2016) or even genomic ("genome dominance") scale (Edger et al. 2017; Yang et al. 2016; Zhang et al. 2015; Flagel and Wendel 2010; Schnable et al. 2011; Garsmeur et al. 2014); the poorly understood phenomenon of "expression level dominance" (Rapp et al. 2009; Yoo et al. 2013; Grover et al. 2012; Zhang et al. 2016; Akhunova et al. 2010; Liu et al. 2014); and the modification of duplicated gene co-expression networks (Gallagher et al. 2016; Hu et al. 2016). A hallmark of these phenomena is deviation from vertical transmission of preexisting patterns, or the "parental legacy", inherited from the two progenitors (Buggs et al. 2014). These deviations collectively represent regulatory novelty that either accompanied or evolved following genome merger and doubling.

Notwithstanding this progress in our understanding of expression alteration accompanying allopolyploidization, there remains a need to develop further an encompassing conceptual framework. Here we propose such a framework based on the characterization of regulatory 
divergence between parental species and subsequent changes at the allopolyploid level. In terms of parental divergence, identifying the types of regulatory changes that have evolved between diploids has long been a focus of classical allele-specific expression (ASE) analysis (Wittkopp et al. 2004). That is, allele-specific expression in F1 hybrids provides a readout of relative cisacting activity in a common trans environment, whereas expression differences between parental species not attributed to cis-acting divergence are inferred to be caused by trans-acting variations. However, how interactions among divergent regulatory alleles affect gene expression in natural hybrid and allopolyploid species remain poorly understood, so uncovering the interplay between the ever-evolving cis and trans elements is critical for understanding phenotypic innovations that emerge following genomic merger and doubling. Here we extend the classical ASE model to the polyploid level, by considering the duplicated sets of cis-trans relationships initiated by interspecific hybridization.

As illustrated in Figure 1A, we use the cotton (Gossypium L.) allopolyploid system as an example, as it is illustrative of many of the model systems used today in studies of polyploidy. Allotetraploid ("AD genome") cottons originated 1-2 million years ago from a hybridization event between two diploid species (“A” and "D”) followed by whole-genome duplication (Wendel and Grover 2015; Wendel and Cronn 2003; Wendel et al. 2010). The descendants of the parental diploid species remain extant (“A2" and "D5"), from which a synthetic F1 hybrid was generated; this has been used to disentangle expression changes due to hybridization from those arising later from polyploidy and subsequent evolution (Yoo et al. 2014; Flagel et al. 2008; Flagel and Wendel 2010). For the synthetic F1 hybrid and natural tetraploid cottons, the expression of each pair of duplicated genes (homoeologs "At" and "Dt", with " $t$ " denoting subgenome) is governed by four sets of cis-trans relationships, including two intra-subgenome interactions derived from each of the parental diploids ( $a a$ and $d d$ ), and two newly formed intersubgenome interactions ( $a d$ and $d a)$.

According to the ASE model (Wittkopp et al. 2004), regulatory divergence acting only in cis between the parental diploids will be mirrored as allele-specific expression in the hybrid and polyploid (At/Dt = A2/D5, where At, Dt, A2 and D5 refer to expression levels for those genic copies; Figure 1B). Any deviations from the parental divergence (i.e., At/Dt $\neq$ A2/D5) can be 
assigned to the influence of trans variation, either acting only in trans (At/Dt $=1$, because of the common trans environment) or by variants acting both in cis and trans (At/Dt $\neq 1$ ). The latter combinatorial effect may also be invisible prior to interspecific hybridization (A2/D5 =1 and $\mathrm{At} / \mathrm{Dt} \neq 1$ ), as cis and trans variants may be compensatory. Such "compensatory" patterns have been suggested to result from stabilizing selection in order to maintain conserved gene expression during parental divergence (Shi et al. 2012; Tirosh et al. 2009), whereas cross interactions between the independently derived genetic variants give rise to immediate expression novelty following genomic merger. In comparison with the "cis only" and "trans only" effects that have been extensively studied in plant hybrid and allopolyploid systems (Shi et al. 2012; Lemmon et al. 2014; Xu et al. 2014; Combes et al. 2015; Chaudhary et al. 2009; Springer and Stupar 2007; Bell et al. 2013; He et al. 2016), understanding the molecular basis of the combinatorial effects of cis and trans variants has been challenging. For example, did the coevolution of cis and trans elements occur in one or both diploid species? How did they evolve from their ancestral states? How do "foreign" (those from different genomes or species) and "native" (from the same genome or species) interactions differ? Is it possible and/or to what extent are "native" and "foreign" interactions preferred? That is, are these relationships asymmetrical? The common trans environment shared by both homoeologs has made these questions conceptually difficult to investigate.

Therefore, let us consider more specifically how trans regulators of different origins act on their cognate and cross-genome targets. For instance, expression of the At homoeolog is determined through its own cis elements interacting with both the A- and D-genome trans factors (represented by $a a+d a$ ), while expression in the diploid parent is attributed to only the cis-trans relationship native to the A-genome diploid $(a a)$. Thus, the contrast between the homoeologspecific expression (At/Dt) and parental expression divergence (A2/D5) can be modeled as:

$$
H_{r}=\log _{2}\left(\frac{\mathrm{At}}{\mathrm{Dt}}\right)-\log _{2}\left(\frac{\mathrm{A} 2}{\mathrm{D} 5}\right)=\log _{2}\left(\frac{a a+d a}{d d+a d}\right)-\log _{2}\left(\frac{a a}{d d}\right)=\log _{2}\left(\frac{1+\frac{d a}{a a}}{1+\frac{a d}{d d}}\right)
$$

where $H_{r}$ represents the impact of hybridization on relative homoeolog expression, and is also equivalent to the additive inverse of the trans effect (i.e., $\left.\log _{2}\left(\frac{\mathrm{At}}{\mathrm{Dt}}\right)-\log _{2}\left(\frac{\mathrm{A} 2}{\mathrm{D} 5}\right)\right)$ estimated in classic ASE analysis (Figure 1B). This acknowledges that hybridization inherently affects 
homoeolog-specific expression in trans, dependent on the relative effects of inter- versus intrasubgenome interactions. Although the foregoing algebraic inference is not substantially different from that of classic ASE analysis, the perspective is nonetheless meaningful. Not only is the impact of hybridization, $H_{r}$, conceptually distinguished from how cis and trans variants contribute to parental divergence, but Eq. 1 also presents a method to quantify how intersubgenome interactions differentially regulate each homoeolog relative to intra-subgenome interactions ( $d a / a a$ vs. $a d / d d)$. As summarized in Figure 1C, the impact of hybridization varies across plant systems, and correlates with the amount of expression divergence between parental species. A histogram of significant $H_{r}$, as exemplified for cotton (Yoo et al. 2013), is indicative of asymmetrical regulation by cross-genome interactions; that is, inter-subgenome interactions have a stronger relative effect on At than Dt. This realization focuses attention on intersubgenome interactions, which are most relevant to gene expression alteration accompanying hybridization per se.

In comparison with the trans action of hybridization per se, how genome doubling alters homoeolog gene expression is complicated by multiple issues of scaling and stoichiometry. With the increase of DNA content accompanying allopolyploidy, imperfect proportionalities and nonlinear relationships with cellular and nuclear volumes set in motion a cascade of stoichiometric imbalances (among, for example, transcriptional machineries and transcription factors), which collectively alter gene expression. Because the physiochemical responses of individual homoeologs vary from gene to gene, it is not yet possible to systematically predict how stoichiometric imbalances triggered by genome merger and doubling will impact regulatory interactions. It does appear, however, that the increased range of homoeolog-specific expression (when the variance of $\frac{\mathrm{At}^{\text {allo }}}{\mathrm{Dt}^{\text {allo }}}$ is larger than $\frac{\mathrm{At}^{\mathrm{F} 1}}{\mathrm{Dt}^{\mathrm{F} 1}}$ ) is expected, as reported in cotton (Yoo et al. 2013), wheat (Wang et al. 2016) and rice (Xu et al. 2014; Sun et al. 2017).

In a cis-trans framework, genome doubling can be modeled by contrasting homoeolog-specific expression between the corresponding allopolyploid and the F1 hybrid. The impact of genome doubling $W_{r}$ is as follows: 


$$
\begin{gathered}
W_{r}=\log _{2}\left(\frac{\mathrm{At}}{\mathrm{Dt} \text { allo }}\right)-\log _{2}\left(\frac{\mathrm{At}^{\mathrm{F} 1}}{\mathrm{Dt}^{\mathrm{F} 1}}\right)=\log _{2}\left(\frac{\widetilde{a a}+\widetilde{d a}}{\widetilde{d d}+\widetilde{a d}}\right)-\log _{2}\left(\frac{a a+d a}{d d+a d}\right) \\
=\log _{2}\left(\frac{\widetilde{a a}+\widetilde{d a}}{a a+d a}\right)-\log _{2}\left(\frac{\widetilde{d d}+\widetilde{a d}}{d d+a d}\right)
\end{gathered}
$$

where the cis-trans interactions in allopolyploids are marked with accent as $\widetilde{a a}, \widetilde{d a}, \widetilde{d d}$ and $\widetilde{a d}$. The same notion applies to the overall effect of allopolyploidization, $P_{r}$ :

$$
\begin{aligned}
& P_{r}=\log _{2}\left(\frac{\text { At }}{\text { allo }} \text { Dt } t^{\text {allo }}\right)-\log _{2}\left(\frac{\mathrm{A} 2}{\mathrm{D} 5}\right)=\log _{2}\left(\frac{\widetilde{a a}+\widetilde{d a}}{\widetilde{d d}+\widetilde{a d}}\right)-\log _{2}\left(\frac{a a}{d d}\right) \\
& =\log _{2}\left(\frac{\widetilde{a a}+\widetilde{d a}}{a a}\right)-\log _{2}\left(\frac{\widetilde{d d}+\widetilde{a d}}{d d}\right)
\end{aligned}
$$

Thus, the emergence of polyploid-specific patterns $\left(W_{r} \neq 0\right.$ and/or $\left.P_{r} \neq 0\right)$ depends on the alteration of any or all cis-trans interactions, which in natural allopolyploids will ensue from a spectrum of stoichiometric responses, epigenetic remodeling and genetic changes.

How these changes collectively affect regulatory interactions are relevant to several of the principal generalizations about gene expression in allopolyploids. For example, under what circumstances do these interactions preferentially shift homoeolog expression ratios towards one progenitor or the other (e.g. more $\frac{\mathrm{At}^{\text {allo }}}{\mathrm{Dt}^{\text {allo }}}>1$ than $\left.\frac{\mathrm{At}^{\text {allo }}}{\mathrm{Dt}^{\text {allo }}}<1\right)$ ? In other words, how might altered cis-trans interactions in allopolyploids account for "genome dominance" (Schnable et al. 2011)? Similarly, how might this perspective shed light on the observation of preferential or biased transcription of one of the two co-resident genomes in an allopolyploid ("unbalanced homoeolog expression bias" at the genomic scale (Grover et al. 2012))?

One possible insight into the mechanisms underlying these dynamics is offered by Bottani et al. (2018), who adapted a kinetics model of transcription factor (TF) binding (Bost and Veitia 2014; Chu et al. 2009) to allopolyploidy. They demonstrated that when cross-regulation is involved, parental difference in TF affinity rather than concentration is the key driver of differential transcription response for homoeologous target genes. Thus, a dominant effect of the parental TF 
that displays higher affinity presents a quantitative explanation for "gene dominance" immediately after allopolyploidy. Next, the authors expanded the simple one-step model of TF binding to two sequential reactions - first the establishment of chromatin accessibility, then transcription factor binding to the accessible promoter site to activate transcription (Figure 1D). This additional epigenetic component thereby provides a testing ground to extend gene-level dominance to the genomic/epigenomic scale. For example, for the parental genome with a larger euchromatic content, more nonfunctional binding sites are accessible, hence creating a higher noise/signal level from the perspective of TF binding. Thus, TF binding affinities and/or concentrations are expected to scale with accessible euchromatin content, all else being equal, in which case, higher TF binding affinity is a likely outcome. As Bottani et al. (2018) showed, when homoeologous TF affinities are proportional to the ratio of euchromatic content, the parental genome with larger euchromatic content is destined to display higher transcription activities following genomic merger and doubling, hence becoming the "dominant" subgenome in allopolyploids.

It is worth nothing that this quantitative model is, to some extent, in line with the prevailing explanation for biased homoeolog expression and biased genome fractionation, based on the "genomic legacy" of TE contents (Wendel et al. 2018; Steige and Slotte 2016). This explanation has been conceptualized in recent years from accumulating literature on chromatin modification, TE content, and small RNA biology (Springer et al. 2016; Diez et al. 2014; Zhang et al. 2017; Renny-Byfield et al. 2017; Yang et al. 2016). Phrased simply, the different parental states of TE load and distribution between sub-genomes lead to differentiated epigenetic control (e.g. small RNA populations and preferential recruitment of epigenetic modifiers) for homoeolog expression, and as a consequence the homoeolog physically closer to epigenetically silenced TEs is more likely to be repressed via localized heterochromatinization, and even lost in the longer term (hence, "biased fractionation"; see recent review by Wendel et al. (2018)).

A key difference between the euchromatin/TF model (Bottani et al. 2018) and the TE model (Wendel et al. 2018; Steige and Slotte 2016), which also makes them complementary to each other, is that the euchromatin/TF model is dependent on a difference in TF affinities and euchromatin content that co-evolved in parental genomes, whereas the TE/biased-fractionation 
model mainly considers differences in chromatin accessibility and gene expression mediated by parental TE adjacency (Figure 1D). What the two models share is the requirement of inheritance of differentiated parental conditions, one being TF affinity while the other is TE adjacency. By analogy to studying the impact of allopolyploidy on homoeolog expression ratios $\left(P_{r}\right)$, as defined above, the effects of inheritance of these parental states can be tested by, with superscripts denoting partitioning of mechanistic effects, $P_{r}^{T F} \cong 0$ for the measure of TF affinity, and $P_{r}^{e p i} \cong$ 0 for TE adjacency and/or epigenetic accessibility. In reality, both scenarios are likely to be intertwined in natural situations, and may even be in conflict with each other. For example, two homoeologs may differ in terms of regulator TF affinity (for whatever reason), but the homoeolog with stronger TF binding may still be expressed at a lower level due to a nearby TE insertion. On the other hand, two homoeologs that differ in promoter accessibility may still be equally expressed, if stronger TF affinity is newly gained for the less accessible homoeolog, or the less accessible promoter has gained more functional binding sites since allopolyploidy. Obviously, a co-examination of both scenarios is most likely to uncover the determinative mechanisms for homoeolog expression divergence.

The foregoing considerations present both a useful cis-trans framework and an experimental agenda for disentangling genetic from epigenetic and higher-order effects that impact gene expression in hybrids and allopolyploids. Of course, translating this conceptual structure into empirical estimates will require more than just expression data, but access to genetic and epigenetic regulatory information is now within reach in many systems. For example, a spectrum of technologies is available to interrogate transcription factor binding to promoters (Bartlett et al. 2017; Jin et al. 2017; Landt et al. 2012; Weirauch et al. 2014), and similarly, a range of chromatin assays (Lane et al. 2014; Celniker et al. 2009; Lu et al. 2017; Jiang 2015; Zentner and Henikoff 2012) are now practical that permit the assessment of the relative accessibility of homoeologs and orthologs to the transcriptional machinery; one was recently applied in maize to connect chromatin states with biased fractionation following ancient polyploid event (RennyByfield et al. 2017). It is the joint application of these technologies with expression data, using the conceptual partitioning described here, which will facilitate new understanding of duplicate gene behavior in hybrids and polyploids. 
In addition to homoeolog-specific expression patterns of expression bias and genome dominance, several key phenomena that characterize novel patterns of aggregated homoeolog expression have also been extensively studied, such as additive and non-additive expression, expressionlevel dominance, and transgressive expression, as reviewed (Yoo et al. 2014). Interpreting these patterns across systems remains an issue due to terminological inconsistency (Grover et al. 2012), among other factors. Perhaps more germane is the point that conceptual relationships among these different phenomena are not well understood, thereby impeding the synthesis required to uncover the underpinnings of duplicate gene expression evolution. The approach outlined here may facilitate such an understanding, by focusing attention on the interplay between genomic legacy features such as TE adjacency and chromatin state, biophysical interactions such as TF binding efficiency, and how these ancestral as well as newly formed cistrans relationships govern expression evolution accompanying genome merger and doubling. As examples, we highlight two broad questions for which our conceptual framework may find utility:

\section{(1) To what extent do homoeolog expression bias and non-additivity reflect novel,} cis/trans interactions? Homoeolog expression bias is when one of two duplicated genes (homoeologs) is expressed more than the other; that is, $\log _{2}\left(\frac{\mathrm{At}}{\mathrm{Dt}}\right) \neq 0$. As modeled in Figure 1A, four sets of inter- and intra-subgenome interactions are involved, and even the parental sets may have been altered following genomic merger and doubling (i.e., $\log _{2}\left(\frac{\widetilde{a a}+\widetilde{d a}}{\widetilde{d d}+\widetilde{a d}}\right) \neq 0$ ). The amount of homoeolog expression bias that resembles parental divergence is relatively consistent among plant species (under 20\%), whereas the amount of expression bias attributed to cross-genome interactions and other types of alterations is more variable (1.4\%-37.8\%); these estimates were extracted from studies of widely diverged plants - arabidopsis (Shi et al. 2012), cotton (Yoo et al. 2013), maize (Lemmon et al. 2014), rice (Xu et al. 2014) and coffee (Combes et al. 2015). Similarly, to test for expression additivity, it is common to compare total expression for a pair of homoeologs $(T=A t+D t)$ to the average of parental expression values $\left(M=\frac{A 2+D 5}{2}\right)$. Because current methods like RNA-seq rely on per-transcriptome normalization to compare expression level across samples, there is an underlying assumption of equal transcriptome size 
(Visger et al. 2017); this, however, likely is not true in most cases (Coate and Doyle 2010) due to the multiple stoichiometric and volumetric cascades that affect gene expression following hybridization and doubling. As shown in Figure 1E, additive expression patterns are determined by the equal total inter- and intra- effects, which has no direct equivalence with any ASE category (Figure 1B). Non-additive expression patterns, including expression-level dominance and transgressive expression levels, arise from all four sets of regulatory interactions, these reflecting complex non-linear biochemical and biophysical interactions. This may help explain the large variation in non-additive expression patterns, ranging from less than $1 \%$ to $7 \%$ in different allohexaploid wheat species (Chelaifa et al. 2013; Chague et al. 2010), from 23 to 61\% among variable cotton tissues (Rambani et al. 2014; Flagel and Wendel 2010; Yoo et al. 2013), and from $42 \%$ to $60 \%$ under two temperature conditions in coffee (Bardil et al. 2011). Teasing apart the mechanistic basis of these novel cis/trans interactions poses an interesting research challenge for future studies.

\section{(2) How is the direction of expression level dominance determined by cis and trans}

regulation? It has been suggested that expression-level dominance toward one parent is mainly caused by up- or down-regulation of the homoeolog of the "less dominant" parent (Shi et al. 2012; Yoo et al. 2013; Cox et al. 2014; Combes et al. 2015). Taking the Adominant expression pattern as an example (Figure 1E, see "A-dominant" row), the joint effect of inter- and intra- interactions approximates the effect of equal number of intra Agenome regulation; if up- or down- regulation is mainly observed for the Dt homoeolog to approach the A-like expression (i.e., $d d+a d=2 a a$ ), the intra- and inter- effects of At are equal to each other (i.e., $d a=a a$ ). This implies that the At expression is mainly determined by its cis element regardless of the origin of trans factors, while at the same time the Dt expression is under strong influence of the At trans factors. Thus, expression level dominance is likely to be associated with divergent trans factors between diploid progenitors, and the progenitor with stronger, more influential trans factors will become dominant with respect to total gene expression. In this context, it will be interesting to explore whether candidate trans factors such as TFs are differentiated between homoeologs in terms of concentrations and affinities. It will also be interesting to 
evaluate whether the strong cis effect of the dominant homoeolog is caused by sequence variation of binding motifs or by a more accessible chromatin formation. Because intersubgenome interactions can up- or down- regulate target homoeologs, the direction of expression level dominance appears not to be associated with the direction of homoeolog bias; it will be interesting to parse the underlying mechanisms of this distinction.

Beyond the gene-centric characterization of expression changes, another relevant and pressing question concerns how gene-to-gene networks are reshaped by genomic merger and doubling, in terms of the genome-wide collection of inter- and intra- subgenome interactions? As recently reviewed by Gallagher et al. (2016), co-expression network analysis in polyploids not only has the potential to facilitate a better understanding of the complex 'omics' underpinnings of phenotypic and ecological traits, but also may provide novel insight into the interaction among duplicated genes and genomes. Given that previous work in allopolyploids (e.g. wheat (Pfeifer et al. 2014) and cotton (Hu et al. 2016)) are mainly based on aggregated co-expression relationships of homoeologs, one future direction is to generate networks considering homoeolog expression separately, thereby allowing the direct evaluation of topological dynamics in terms of gain and loss of intra- and inter-subgenome relationships (Conant 2010; Conant and Wolfe 2008; Conant and Wolfe 2006). Although co-expression relationships do not necessarily represent physical interactions between cis and trans regulatory elements, the gene-to-gene interconnections that are inferred based on the "guilt-by-association" principle provide an alternative, and parallel approach to estimate the impact of genomic merger and doubling, under the same analytic framework as proposed. Future analyses of gene networks could include integration with parental cis-trans divergence, novel cross-genome interactions, and various expression-level phenomena, together with other epigenetic and physiochemical datasets.

In conclusion, the opportunity to advance our understanding of transcriptome dynamics in hybrids and allopolyploids is being enabled by the maturation of multiple "omics" technologies and conceptual advances, the latter including a focus on the mechanistic underpinnings of intergenomic cis-trans interactions, as explicated here. It is likely that these perspectives and approaches will yield new insight into the origin of physiological and phenotypic responses to hybridization and polyploidy, and thereby to the evolutionary process in general. 


\section{Acknowledgements}

We thank Corrinne E. Grover and Justin Conover for helpful discussion and comments on the manuscript. We are also grateful for comments and criticisms from the reviewers and editors, which prompted us to revise and substantially improve this manuscript. Research in the Wendel laboratory has been funded by NSF, whose support we gratefully acknowledge. 
A
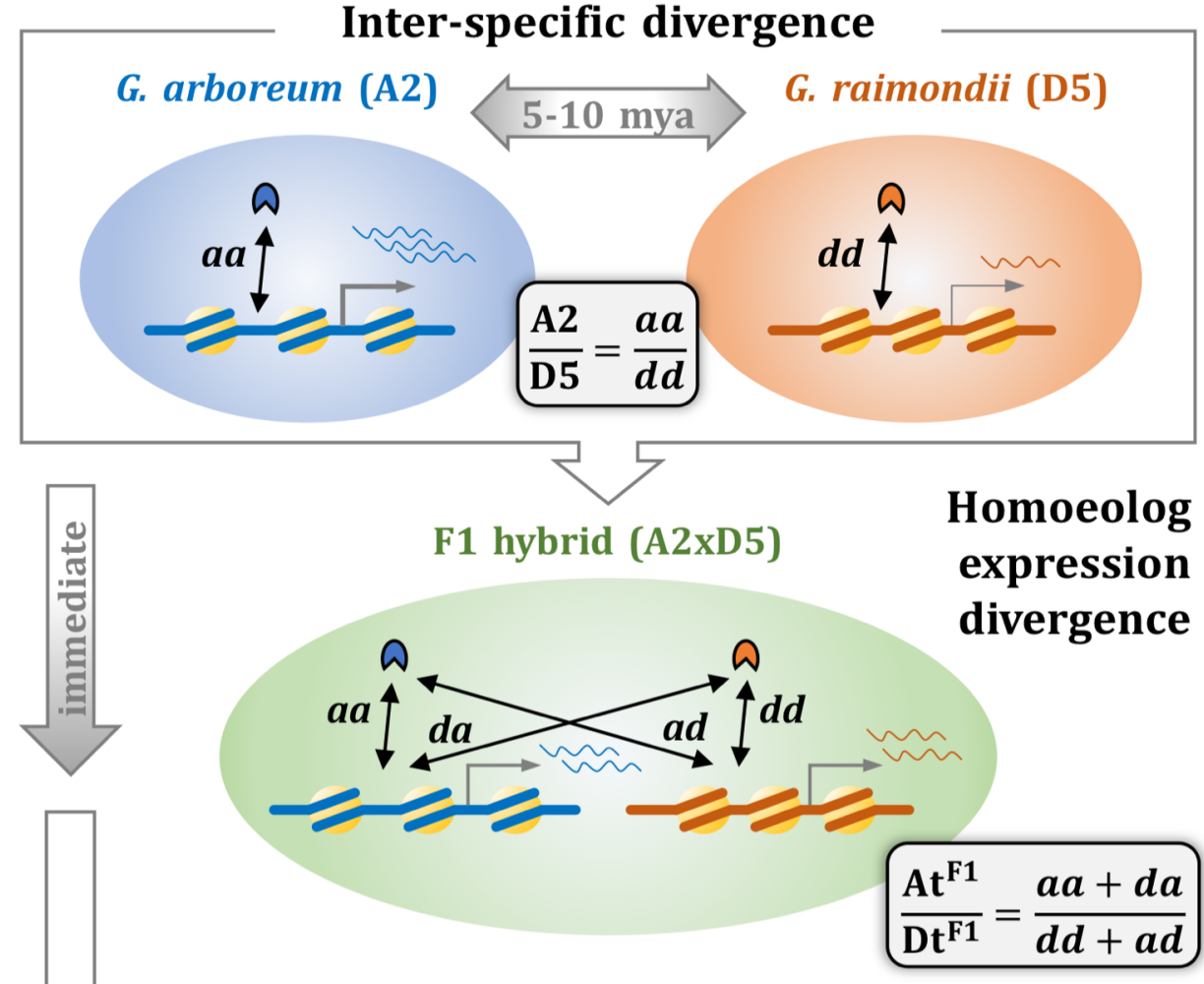

Natural Allopolyploid

G. hirsutum (AD1)
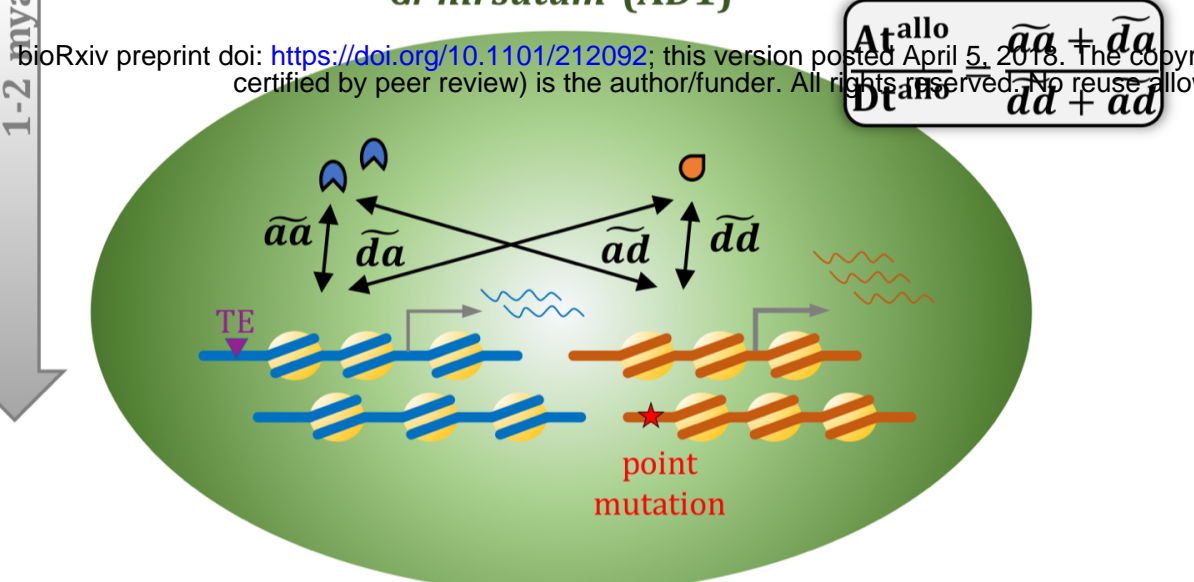

B

\section{Allele-Specific Analysis (ASE)}

cis + trans: $\boldsymbol{A}=\log _{2}\left(\frac{\mathrm{A} 2}{\mathrm{D} 5}\right) \quad$ cis: $\boldsymbol{B}=\log _{2}\left(\frac{\mathrm{At}}{\mathrm{Dt}}\right) \quad$ trans: $\boldsymbol{A}-\boldsymbol{B}$

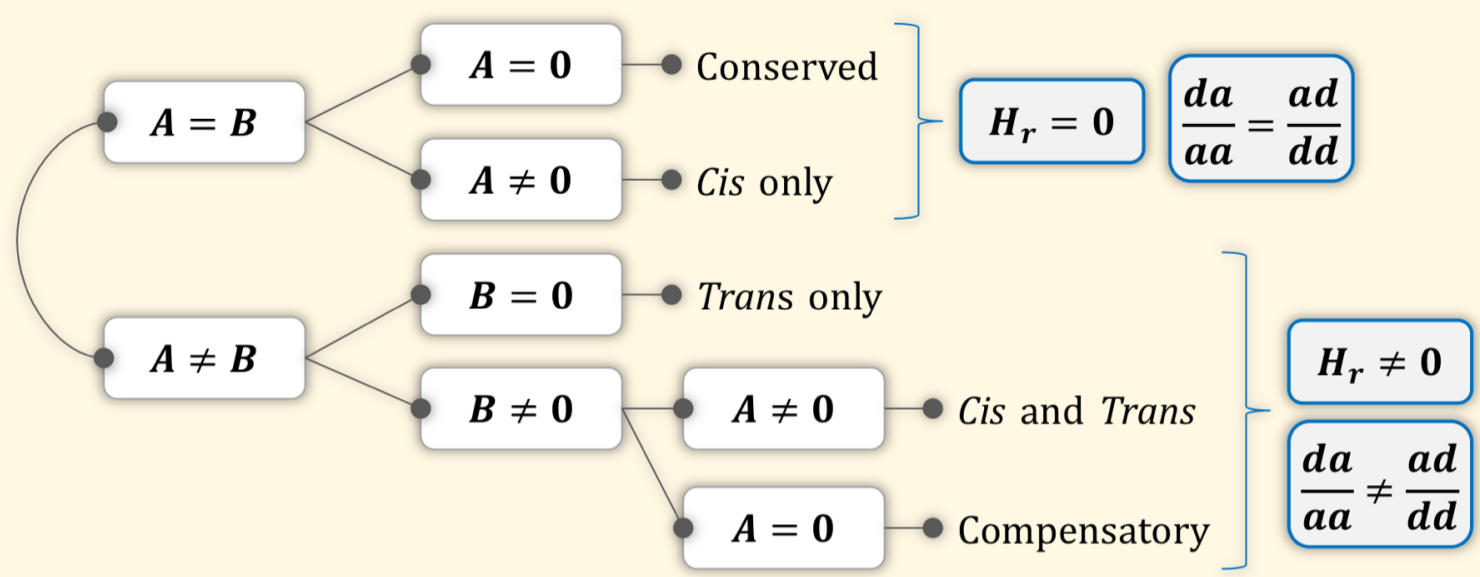

C

\begin{tabular}{|c|c|c|c|c|c|c|}
\hline $\begin{array}{l}\text { prespipt.(phish v } \\
\text { sion? }\end{array}$ & $\begin{array}{l}\text { Parental } \\
\text { divergence } \\
{ }^{\circ o t}(\boldsymbol{A} \neq \mathbf{0})\end{array}$ & $\begin{array}{c}\text { Homoeolog } \\
\text { bias } \\
(\boldsymbol{B} \neq \mathbf{0})\end{array}$ & $\begin{array}{c}\text { Hybridizatior } \\
\text { impact } \\
\left(\boldsymbol{H}_{\boldsymbol{r}} \neq \mathbf{0}\right)\end{array}$ & & $\begin{array}{r}\boldsymbol{H}_{\boldsymbol{r}} \text { indic } \\
\text { inter-/in } \\
\text { between }\end{array}$ & \\
\hline arabidopsis & $31.7 \%$ & $23.9 \%$ & $12.8 \%$ & 8 & e.g. cotton & \\
\hline rice & $37.7 \%$ & $28.0 \%$ & $29.7 \%$ & 8 & & \\
\hline maize & $60.8 \%$ & $51.1 \%$ & $50.8 \%$ & $\frac{9}{5}$ & & \\
\hline coffee & $51.4 \%$ & $33.0 \%$ & $35.9 \%$ & gg & & \\
\hline cotton & $10.1 \%$ & $8.7 \%$ & $3.7 \%$ & 0 & & \\
\hline
\end{tabular}

* Data extracted from arabidopsis (Shi et al. 2012), rice (Xu et al. 2014), maize (Lemmonet al. 2014) coffee (Combes et al. 2015), and cotton (Yoo et al., 2013).

D

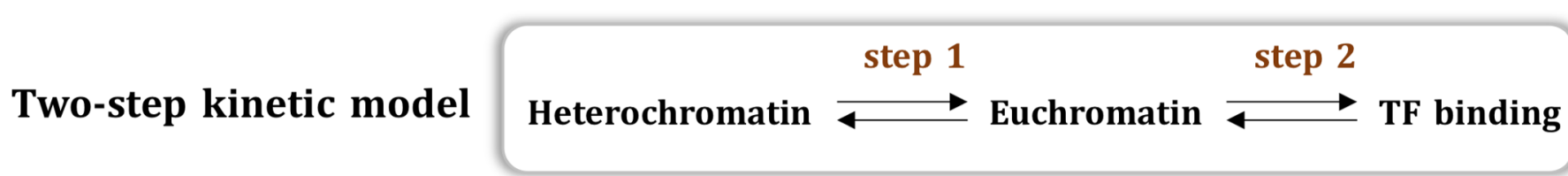

Measurement

"Genome dominance"
TE distribution, epigenetic accessibility, etc.

TE model: $P_{r}^{e p i} \cong 0$
Euchromatin/TF model: $P_{r}^{T F} \cong 0$
$\mathrm{TF}$ concentration and affinity

\section{Transcription}

Diploid progenitors

$\llbracket \boldsymbol{P}_{r}\left(\boldsymbol{H}_{r}, \boldsymbol{W}_{r}\right)$

Allopolyploids

Aggregated homoeolog expression patterns - Additivity, dominance, and transgressive expression

\begin{tabular}{lcc} 
Expression description & Measure & Model \\
\hline Total expression $(\mathrm{T})$ & $\mathrm{T}=\mathrm{At}+\mathrm{Dt}$ & $(a a+d a+d d+a d) / 2$ \\
Parental A-genome expression & $\mathrm{A} 2$ & $2 a a$ \\
Parental D-genome expression & $\mathrm{D} 5$ & $2 d d$ \\
Mid-parental expression $(\mathrm{M})$ & $(\mathrm{A} 2+\mathrm{D} 5) / 2$ & $a a+d d$
\end{tabular}

\begin{tabular}{|l|l|l|l|}
\hline Class & Hypothesis & Analytic model & Interpretation \\
\hline Additivity & $\mathrm{T}=\mathrm{M}$ & $d a+a d=a a+d d$ & inter- equals to intra- effects \\
\hline $\begin{array}{l}\text { Transgressive } \\
\text { expression }\end{array}$ & $\begin{array}{l}\mathrm{T}>\max (\mathrm{A} 2, \mathrm{D} 5) \text { or } \\
\mathrm{T}<\min (\mathrm{A} 2, \mathrm{D} 5)\end{array}$ & $\begin{array}{l}d a+a d>a a+d d, \text { or } \\
d a+a d<a a+d d\end{array}$ & $\begin{array}{l}\text { inter- NOT equal to intra- } \\
\text { effects }\end{array}$ \\
\cline { 1 - 3 } A-dominant & $\mathrm{T}=\mathrm{A} 2$ & $d a+a d+a a+d d=4 a a \& a a \neq d d$ & $\begin{array}{l}\text { Asymmetric inter-effects } \\
\text { have been consistently } \\
\text { reported. }\end{array}$ \\
\hline D-dominant & $\mathrm{T}=\mathrm{D} 5$ & $d a+a d+a a+d d=4 d d \& a a \neq d d$ & \\
\hline
\end{tabular}


Figure 1. A proposed analytical framework for understanding regulatory novelty accompanying hybridization and allopolyploidy, using the cotton (Gossypium L.) allopolyploid system as an example.

(A) Between the parental diploid species G. arboreum (A2) and G. raimondii (D5), differential gene expression and/or chromatin accessibility are determined by the divergence of corresponding intra-genome cis-trans interactions $a a$ and $d d$, respectively. Following genomic merger and doubling, the At and Dt homoeolog divergence is governed by two more sets of newly formed inter-subgenome interactions $a d$ and $d a$ (first letter indicates trans origin, and second letter indicates cis origin). In natural allopolyploids, stoichiometric changes and sequence evolution (e.g. TE insertion and point mutation) may further alter cis-trans interactions to become $\widetilde{a a}, \widetilde{d a}, \widetilde{d d}$ and $\widetilde{a d}$.

(B) A schematic diagram of classic allele-specific expression analysis (ASE), with interpretations based on relative inter- versus intra- cis-trans interactions noted in blue boxes. (C) Percentages of parental divergence, homoeolog expression bias and hybridization impact $\left(H_{r}\right)$ in various plant systems. The histogram of significant $H_{r}$ is shown for cotton.

(D) According to a two-step kinetic model of gene transcription, two hypotheses of "genome dominance", the euchromatin/TF model (Bottani et al. 2018) and the TE model (Wendel et al. 2018; Steige and Slotte 2016), are complementary to each other. These provide a conceptual framework for revealing the mechanisms that underlie novel cis-trans interactions.

(E) Understanding non-additive expression patterns as regulated by cis-trans interactions. 


\section{References}

Akama S, Shimizu-Inatsugi R, Shimizu KK, Sese J (2014) Genome-wide quantification of homeolog expression ratio revealed nonstochastic gene regulation in synthetic allopolyploid Arabidopsis. Nucleic Acids Res 42 (6):e46. doi:10.1093/nar/gkt1376

Akhunova AR, Matniyazov RT, Liang H, Akhunov ED (2010) Homoeolog-specific transcriptional bias in allopolyploid wheat. BMC genomics 11:505. doi:10.1186/14712164-11-505

Anssour S, Krugel T, Sharbel TF, Saluz HP, Bonaventure G, Baldwin IT (2009) Phenotypic, genetic and genomic consequences of natural and synthetic polyploidization of Nicotiana attenuata and Nicotiana obtusifolia. Ann Bot 103 (8):1207-1217. doi:10.1093/aob/mcp058

Bardil A, de Almeida JD, Combes MC, Lashermes P, Bertrand B (2011) Genomic expression dominance in the natural allopolyploid Coffea arabica is massively affected by growth temperature. New Phytol 192 (3):760-774. doi:10.1111/j.1469-8137.2011.03833.x

Bartlett A, O'Malley RC, Huang SC, Galli M, Nery JR, Gallavotti A, Ecker JR (2017) Mapping genome-wide transcription-factor binding sites using DAP-seq. Nature protocols 12 (8):1659-1672. doi:10.1038/nprot.2017.055

Bell GD, Kane NC, Rieseberg LH, Adams KL (2013) RNA-seq analysis of allele-specific expression, hybrid effects, and regulatory divergence in hybrids compared with their parents from natural populations. Genome biology and evolution 5 (7):1309-1323. doi:10.1093/gbe/evt072

Bost B, Veitia RA (2014) Dominance and interloci interactions in transcriptional activation cascades: models explaining compensatory mutations and inheritance patterns. Bioessays 36 (1):84-92. doi:10.1002/bies.201300109

Bottani S, Zabet NR, Wendel JF, Veitia RA (2018) Gene expression dominance in allopolyploids: hypotheses and models. Trends Plant Sci. doi:10.1016/j.tplants.2018.01.002

Bottley A (2014) Epigenetic variation amongst polyploidy crop species. In: Epigenetics in Plants of Agronomic Importance: Fundamentals and Applications. Springer International Publishing, pp 33-46. doi:10.1007/978-3-319-07971-4_3

Buggs RJ, Doust AN, Tate JA, Koh J, Soltis K, Feltus FA, Paterson AH, Soltis PS, Soltis DE (2009) Gene loss and silencing in Tragopogon miscellus (Asteraceae): comparison of natural and synthetic allotetraploids. Heredity 103 (1):73-81. doi:10.1038/hdy.2009.24

Buggs RJ, Wendel JF, Doyle JJ, Soltis DE, Soltis PS, Coate JE (2014) The legacy of diploid progenitors in allopolyploid gene expression patterns. Philosophical transactions of the Royal Society of London Series B, Biological sciences 369 (1648):20130354. doi:10.1098/rstb.2013.0354

Celniker SE, Dillon LA, Gerstein MB, Gunsalus KC, Henikoff S, Karpen GH, Kellis M, Lai EC, Lieb JD, MacAlpine DM, Micklem G, Piano F, Snyder M, Stein L, White KP, Waterston RH, mod EC (2009) Unlocking the secrets of the genome. Nature 459 (7249):927-930. doi:10.1038/459927a

Chague V, Just J, Mestiri I, Balzergue S, Tanguy AM, Huneau C, Huteau V, Belcram H, Coriton O, Jahier J, Chalhoub B (2010) Genome-wide gene expression changes in genetically stable synthetic and natural wheat allohexaploids. New Phytol 187 (4):1181-1194. doi:10.1111/j.1469-8137.2010.03339.x 
Chaudhary B, Flagel L, Stupar RM, Udall JA, Verma N, Springer NM, Wendel JF (2009) Reciprocal silencing, transcriptional bias and functional divergence of homeologs in polyploid cotton (gossypium). Genetics 182 (2):503-517.

doi:10.1534/genetics.109.102608

Chelaifa H, Chague V, Chalabi S, Mestiri I, Arnaud D, Deffains D, Lu Y, Belcram H, Huteau V, Chiquet J, Coriton O, Just J, Jahier J, Chalhoub B (2013) Prevalence of gene expression additivity in genetically stable wheat allohexaploids. New Phytol 197 (3):730-736. doi:10.1111/nph.12108

Chen ZJ (2007) Genetic and epigenetic mechanisms for gene expression and phenotypic variation in plant polyploids. Annu Rev Plant Biol 58:377-406. doi:10.1146/annurev.arplant.58.032806.103835

Chu D, Zabet NR, Mitavskiy B (2009) Models of transcription factor binding: sensitivity of activation functions to model assumptions. J Theor Biol 257 (3):419-429. doi:10.1016/j.jtbi.2008.11.026

Coate JE, Doyle JJ (2010) Quantifying whole transcriptome size, a prerequisite for understanding transcriptome evolution across species: an example from a plant allopolyploid. Genome biology and evolution 2:534-546. doi:10.1093/gbe/evq038

Combes M-C, Dereeper A, Severac D, Bertrand Bi, Lashermes P (2013) Contribution of subgenomes to the transcriptome and their intertwined regulation in the allopolyploid Coffea arabica grown at contrasted temperatures. New Phytologist 200 (1):251-260

Combes M-C, Hueber Y, Dereeper A, Rialle S, Herrera J-C, Lashermes P (2015) Regulatory divergence between parental alleles determines gene expression patterns in hybrids. Genome biology and evolution 7 (4):1110-1121

Conant GC (2010) Rapid reorganization of the transcriptional regulatory network after genome duplication in yeast. Proceedings of the Royal Society of London B: Biological Sciences 277 (1683):869-876

Conant GC, Wolfe KH (2006) Functional partitioning of yeast co-expression networks after genome duplication. PLoS biology 4 (4):e109-e109

Conant GC, Wolfe KH (2008) Turning a hobby into a job: how duplicated genes find new functions. Nat Rev Genet 9 (12):938-950. doi:10.1038/nrg2482

Cox MP, Dong T, Shen G, Dalvi Y, Scott DB, Ganley ARD (2014) An interspecific fungal hybrid reveals cross-kingdom rules for allopolyploid gene expression patterns. PLoS Genet 10 (3):e1004180-e1004180

Diez CM, Roessler K, Gaut BS (2014) Epigenetics and plant genome evolution. Curr Opin Plant Biol 18:1-8. doi:10.1016/j.pbi.2013.11.017

Edger PP, Smith RD, McKain MR, Cooley AM, Vallejo-Marin M, Yuan Y-W, Bewick AJ, Ji L, Platts AE, Bowman MJ, others (2017) Subgenome dominance in an interspecific hybrid, synthetic allopolyploid, and a 140-year-old naturally established neo-allopolyploid monkeyflower. The Plant Cell Online:tpc-00010

Eilam T, Anikster Y, Millet E, Manisterski J, Feldman M (2009) Genome size in natural and synthetic autopolyploids and in a natural segmental allopolyploid of several Triticeae species. Genome 52 (3):275-285. doi:10.1139/G09-004

Flagel L, Udall J, Nettleton D, Wendel J (2008) Duplicate gene expression in allopolyploid Gossypium reveals two temporally distinct phases of expression evolution. BMC Biol 6:16. doi:10.1186/1741-7007-6-16 
Flagel LE, Wendel JF (2010) Evolutionary rate variation, genomic dominance and duplicate gene expression evolution during allotetraploid cotton speciation. The New phytologist 186 (1):184-193. doi:10.1111/j.1469-8137.2009.03107.x

Freeling M, Woodhouse MR, Subramaniam S, Turco G, Lisch D, Schnable JC (2012) Fractionation mutagenesis and similar consequences of mechanisms removing dispensable or less-expressed DNA in plants. Curr Opin Plant Biol 15 (2):131-139. doi:10.1016/j.pbi.2012.01.015

Fulnecek J, Matyasek R, Kovarik A (2009) Faithful inheritance of cytosine methylation patterns in repeated sequences of the allotetraploid tobacco correlates with the expression of DNA methyltransferase gene families from both parental genomes. Mol Genet Genomics 281 (4):407-420. doi:10.1007/s00438-008-0420-8

Gallagher JP, Grover CE, Hu G, Wendel JF (2016) Insights into the ecology and evolution of polyploid plants through network analysis. Molecular ecology 25 (11):2644-2660. doi: $10.1111 / \mathrm{mec} .13626$

Garsmeur O, Schnable JC, Almeida A, Jourda C, D'Hont A, Freeling M (2014) Two evolutionarily distinct classes of paleopolyploidy. Molecular biology and evolution 31 (2):448-454. doi:10.1093/molbev/mst230

Grover CE, Gallagher JP, Szadkowski EP, Yoo MJ, Flagel LE, Wendel JF (2012) Homoeolog expression bias and expression level dominance in allopolyploids. The New phytologist 196 (4):966-971. doi:10.1111/j.1469-8137.2012.04365.x

He F, Arce AL, Schmitz G, Koornneef M, Novikova P, Beyer A, de Meaux J (2016) The footprint of polygenic adaptation on stress-responsive cis-regulatory divergence in the Arabidopsis genus. Mol Biol Evol 33 (8):2088-2101. doi:10.1093/molbev/msw096

Hu G, Hovav R, Grover CE, Faigenboim-Doron A, Kadmon N, Page JT, Udall JA, Wendel JF (2016) Evolutionary conservation and divergence of gene coexpression networks in Gossypium (cotton) seeds. Genome Biol Evol 8 (12):3765-3783. doi:10.1093/gbe/evw280

Jackson SA (2017) Epigenomics: dissecting hybridization and polyploidization. Genome biology 18 (1):117. doi:10.1186/s13059-017-1254-7

Jiang J (2015) The 'dark matter' in the plant genomes: non-coding and unannotated DNA sequences associated with open chromatin. Current opinion in plant biology 24:17-23

Jin J, Tian F, Yang D-C, Meng Y-Q, Kong L, Luo J, Gao G (2017) PlantTFDB 4.0: toward a central hub for transcription factors and regulatory interactions in plants. Nucleic acids research 45 (D1):D1040-D1045

Kawakami T, Strakosh SC, Zhen Y, Ungerer MC (2010) Different scales of Ty1/copia-like retrotransposon proliferation in the genomes of three diploid hybrid sunflower species. Heredity (Edinb) 104 (4):341-350. doi:10.1038/hdy.2009.182

Koh J, Soltis PS, Soltis DE (2010) Homeolog loss and expression changes in natural populations of the recently and repeatedly formed allotetraploid Tragopogon mirus (Asteraceae). BMC genomics 11:97. doi:10.1186/1471-2164-11-97

Kovarik A, Dadejova M, Lim YK, Chase MW, Clarkson JJ, Knapp S, Leitch AR (2008) Evolution of rDNA in Nicotiana allopolyploids: a potential link between rDNA homogenization and epigenetics. Ann Bot 101 (6):815-823. doi:10.1093/aob/mcn019

Landt SG, Marinov GK, Kundaje A, Kheradpour P, Pauli F, Batzoglou S, Bernstein BE, Bickel P, Brown JB, Cayting P, Chen Y, DeSalvo G, Epstein C, Fisher-Aylor KI, Euskirchen G, Gerstein M, Gertz J, Hartemink AJ, Hoffman MM, Iyer VR, Jung YL, Karmakar S, 
Kellis M, Kharchenko PV, Li Q, Liu T, Liu XS, Ma L, Milosavljevic A, Myers RM, Park PJ, Pazin MJ, Perry MD, Raha D, Reddy TE, Rozowsky J, Shoresh N, Sidow A, Slattery M, Stamatoyannopoulos JA, Tolstorukov MY, White KP, Xi S, Farnham PJ, Lieb JD, Wold BJ, Snyder M (2012) ChIP-seq guidelines and practices of the ENCODE and modENCODE consortia. Genome Res 22 (9):1813-1831. doi:10.1101/gr.136184.111

Lane AK, Niederhuth CE, Ji L, Schmitz RJ (2014) pENCODE: a plant encyclopedia of DNA elements. Annu Rev Genet 48:49-70

Leitch AR, Leitch IJ (2008) Genomic plasticity and the diversity of polyploid plants. Science 320 (5875):481-483. doi:10.1126/science.1153585

Lemmon ZH, Bukowski R, Sun Q, Doebley JF (2014) The role of cis regulatory evolution in maize domestication. PLoS genetics 10 (11):e1004745.

doi:10.1371/journal.pgen. 1004745

Levin DA (1983) Polyploidy and novelty in flowering plants. The American Naturalist 122 (1): $1-25$

Liu S, Liu Y, Yang X, Tong C, Edwards D, Parkin IA, Zhao M, Ma J, Yu J, Huang S, Wang X, Wang J, Lu K, Fang Z, Bancroft I, Yang TJ, Hu Q, Wang X, Yue Z, Li H, Yang L, Wu J, Zhou Q, Wang W, King GJ, Pires JC, Lu C, Wu Z, Sampath P, Wang Z, Guo H, Pan S, Yang L, Min J, Zhang D, Jin D, Li W, Belcram H, Tu J, Guan M, Qi C, Du D, Li J, Jiang L, Batley J, Sharpe AG, Park BS, Ruperao P, Cheng F, Waminal NE, Huang Y, Dong C, Wang L, Li J, Hu Z, Zhuang M, Huang Y, Huang J, Shi J, Mei D, Liu J, Lee TH, Wang J, Jin H, Li Z, Li X, Zhang J, Xiao L, Zhou Y, Liu Z, Liu X, Qin R, Tang X, Liu W, Wang Y, Zhang Y, Lee J, Kim HH, Denoeud F, Xu X, Liang X, Hua W, Wang X, Wang J, Chalhoub B, Paterson AH (2014) The Brassica oleracea genome reveals the asymmetrical evolution of polyploid genomes. Nat Commun 5:3930. doi:10.1038/ncomms4930

Lu Z, Hofmeister BT, Vollmers C, DuBois RM, Schmitz RJ (2017) Combining ATAC-seq with nuclei sorting for discovery of cis-regulatory regions in plant genomes. Nucleic acids research 45 (6):e41-e41

Madlung A (2013) Polyploidy and its effect on evolutionary success: old questions revisited with new tools. Heredity 110 (2):99-104. doi:10.1038/hdy.2012.79

Madlung A, Masuelli RW, Watson B, Reynolds SH, Davison J, Comai L (2002) Remodeling of DNA methylation and phenotypic and transcriptional changes in synthetic Arabidopsis allotetraploids. Plant physiology 129 (2):733-746. doi:10.1104/pp.003095

Mirzaghaderi G, Mason AS (2017) Revisiting pivotal-differential genome evolution in wheat. Trends Plant Sci 22 (8):674-684. doi:10.1016/j.tplants.2017.06.003

Parisod C, Alix K, Just J, Petit M, Sarilar V, Mhiri C, Ainouche M, Chalhoub B, Grandbastien MA (2010) Impact of transposable elements on the organization and function of allopolyploid genomes. The New phytologist 186 (1):37-45. doi:10.1111/j.14698137.2009.03096.x

Pfeifer M, Kugler KG, Sandve SR, Zhan B, Rudi H, Hvidsten TR, International Wheat Genome Sequencing C, Mayer KF, Olsen OA (2014) Genome interplay in the grain transcriptome of hexaploid bread wheat. Science 345 (6194):1250091. doi:10.1126/science.1250091

Rambani A, Page JT, Udall JA (2014) Polyploidy and the petal transcriptome of Gossypium. BMC Plant Biol 14:3. doi:10.1186/1471-2229-14-3

Ramsey J, Schemske DW (2002) Neopolyploidy in flowering plants. Annual Review of Ecology and Systematics 33 (1):589-639. doi:10.1146/annurev.ecolsys.33.010802.150437 
Rapp RA, Udall JA, Wendel JF (2009) Genomic expression dominance in allopolyploids. BMC Biol 7:18. doi:10.1186/1741-7007-7-18

Rapp RA, Wendel JF (2005) Epigenetics and plant evolution. The New phytologist 168 (1):8191. doi:10.1111/j.1469-8137.2005.01491.x

Renny-Byfield S, Rodgers-Melnick E, Ross-Ibarra J (2017) Gene fractionation and function in the ancient subgenomes of maize. Mol Biol Evol 34 (8):1825-1832. doi: $10.1093 / \mathrm{molbev} / \mathrm{msx} 121$

Salmon A, Ainouche ML, Wendel JF (2005) Genetic and epigenetic consequences of recent hybridization and polyploidy in Spartina (Poaceae). Molecular ecology 14 (4):11631175. doi:10.1111/j.1365-294X.2005.02488.x

Schnable JC, Springer NM, Freeling M (2011) Differentiation of the maize subgenomes by genome dominance and both ancient and ongoing gene loss. Proceedings of the National Academy of Sciences of the United States of America 108 (10):4069-4074. doi:Doi 10.1073/Pnas. 1101368108

Senerchia N, Felber F, Parisod C (2015) Genome reorganization in F1 hybrids uncovers the role of retrotransposons in reproductive isolation. Proc Biol Sci 282 (1804):20142874. doi:10.1098/rspb.2014.2874

Shcherban AB, Badaeva ED, Amosova AV, Adonina IG, Salina EA (2008) Genetic and epigenetic changes of rDNA in a synthetic allotetraploid, Aegilops sharonensis $\mathrm{x}$ Ae. umbellulata. Genome 51 (4):261-271. doi:10.1139/G08-006

Shi X, Ng DW, Zhang C, Comai L, Ye W, Chen ZJ (2012) Cis- and trans-regulatory divergence between progenitor species determines gene-expression novelty in Arabidopsis allopolyploids. Nat Commun 3:950. doi:10.1038/ncomms1954

Soltis PS, Liu X, Marchant DB, Visger CJ, Soltis DE (2014) Polyploidy and novelty: Gottlieb's legacy. Phil Trans R Soc B 369 (1648):20130351-20130351

Soltis PS, Soltis DE (2016) Ancient WGD events as drivers of key innovations in angiosperms. Curr Opin Plant Biol 30:159-165. doi:10.1016/j.pbi.2016.03.015

Song Q, Zhang T, Stelly DM, Chen ZJ (2017) Epigenomic and functional analyses reveal roles of epialleles in the loss of photoperiod sensitivity during domestication of allotetraploid cottons. Genome biology 18 (1):99-99

Springer NM, Lisch D, Li Q (2016) Creating order from chaos: epigenome dynamics in plants with complex genomes. Plant Cell 28 (2):314-325. doi:10.1105/tpc.15.00911

Springer NM, Stupar RM (2007) Allele-specific expression patterns reveal biases and embryospecific parent-of-origin effects in hybrid maize. Plant Cell 19 (8):2391-2402. doi: $10.1105 /$ tpc. 107.052258

Stebbins GL (1940) The significance of polyploidy in plant evolution. The American Naturalist 74 (750):54-66

Steige KA, Slotte T (2016) Genomic legacies of the progenitors and the evolutionary consequences of allopolyploidy. Curr Opin Plant Biol 30:88-93. doi:10.1016/j.pbi.2016.02.006

Sun Y, Wu Y, Yang C, Sun S, Lin X, Liu L, Xu C, Wendel JF, Gong L, Liu B (2017) Segmental allotetraploidy generates extensive homoeologous expression rewiring and phenotypic diversity at the population level in rice. Mol Ecol 26 (20):5451-5466. doi:10.1111/mec. 14297

Szadkowski E, Eber F, Huteau V, Lode M, Huneau C, Belcram H, Coriton O, ManzanaresDauleux MJ, Delourme R, King GJ, Chalhoub B, Jenczewski E, Chevre AM (2010) The 
first meiosis of resynthesized Brassica napus, a genome blender. The New phytologist 186 (1):102-112. doi:10.1111/j.1469-8137.2010.03182.x

Tate JA, Joshi P, Soltis KA, Soltis PS, Soltis DE (2009) On the road to diploidization? Homoeolog loss in independently formed populations of the allopolyploid Tragopogon miscellus (Asteraceae). BMC plant biology 9:80. doi:10.1186/1471-2229-9-80

Tirosh I, Reikhav S, Levy AA, Barkai N (2009) A yeast hybrid provides insight into the evolution of gene expression regulation. Science 324 (5927):659-662

Van de Peer Y, Maere S, Meyer A (2009) The evolutionary significance of ancient genome duplications. Nat Rev Genet 10 (10):725-732. doi:10.1038/nrg2600

Van de Peer Y, Mizrachi E, Marchal K (2017) The evolutionary significance of polyploidy. Nat Rev Genet 18 (7):411-424. doi:10.1038/nrg.2017.26

Visger C, Wong GK-S, Zhang Y, Soltis PS, Soltis DE (2017) Divergent gene expression levels between diploid and autotetraploid Tolmiea (Saxifragaceae) relative to the total transcriptome, the cell, and biomass. bioRxiv

Wang X, Zhang H, Li Y, Zhang Z, Li L, Liu B (2016) Transcriptome asymmetry in synthetic and natural allotetraploid wheats, revealed by RNA-sequencing. The New phytologist 209 (3):1264-1277. doi:10.1111/nph.13678

Wang X, Zhang Z, Fu T, Hu L, Xu C, Gong L, Wendel JF, Liu B (2017) Gene-body CG methylation and divergent expression of duplicate genes in rice. Scientific reports 7 (1):2675. doi:10.1038/s41598-017-02860-4

Weirauch MT, Yang A, Albu M, Cote AG, Montenegro-Montero A, Drewe P, Najafabadi HS, Lambert SA, Mann I, Cook K, Zheng H, Goity A, van Bakel H, Lozano JC, Galli M, Lewsey MG, Huang E, Mukherjee T, Chen X, Reece-Hoyes JS, Govindarajan S, Shaulsky G, Walhout AJM, Bouget FY, Ratsch G, Larrondo LF, Ecker JR, Hughes TR (2014) Determination and inference of eukaryotic transcription factor sequence specificity. Cell 158 (6):1431-1443. doi:10.1016/j.cell.2014.08.009

Wendel FJ, Doyle JJ (2005) Polyploidy and evolution in plants. In: Henry RJ (ed) Plant diversity and evolution: genotypic and phenotypic variation in higher plants. CABI Publishing, Wallingford, UK, pp 97-117. doi:10.1079/9780851999043.0097

Wendel JF, Brubaker CL, Seelanan T (2010) The origin and evolution of Gossypium. In: Stewart JM, Oosterhuis DM, Heitholt JJ, Mauney JR (eds) Physiology of Cotton. Springer Netherlands, pp 1-18. doi:10.1007/978-90-481-3195-2_1

Wendel JF, Cronn RC (2003) Polyploidy and the evolutionary history of cotton. Advances in Agronomy 78:139-186

Wendel JF, Grover CE (2015) Taxonomy and evolution of the cotton genus, Gossypium. In: Fang DD, Percy RG (eds) Cotton. Agronomy Monograph, vol 57, 2nd edn. American Society of Agronomy, Madison, WI, pp 25-42. doi:10.2134/agronmonogr57.2013.0020

Wendel JF, Lisch D, Hu G, Mason AS (2018) The long and short of doubling down: polyploidy, epigenetics, and the temporal dynamics of genome fractionation. Curr Opin Genet Dev 49:1-7. doi:10.1016/j.gde.2018.01.004

Wittkopp PJ, Haerum BK, Clark AG (2004) Evolutionary changes in cis and trans gene regulation. Nature 430 (6995):85-88. doi:10.1038/nature02698

Xu C, Bai Y, Lin X, Zhao N, Hu L, Gong Z, Wendel JF, Liu B (2014) Genome-wide disruption of gene expression in allopolyploids but not hybrids of rice subspecies. Molecular biology and evolution 31 (5):1066-1076. doi:10.1093/molbev/msu085 
Yang J, Liu D, Wang X, Ji C, Cheng F, Liu B, Hu Z, Chen S, Pental D, Ju Y, Yao P, Li X, Xie K, Zhang J, Wang J, Liu F, Ma W, Shopan J, Zheng H, Mackenzie SA, Zhang M (2016) The genome sequence of allopolyploid Brassica juncea and analysis of differential homoeolog gene expression influencing selection. Nat Genet 48 (10):1225-1232. doi:10.1038/ng.3657

Yoo MJ, Liu X, Pires JC, Soltis PS, Soltis DE (2014) Nonadditive gene expression in polyploids. Annual review of genetics 48:485-517. doi:10.1146/annurev-genet-120213-092159

Yoo MJ, Szadkowski E, Wendel JF (2013) Homoeolog expression bias and expression level dominance in allopolyploid cotton. Heredity 110 (2):171-180. doi:10.1038/hdy.2012.94

Yoo MJ, Wendel JF (2014) Comparative evolutionary and developmental dynamics of the cotton (Gossypium hirsutum) fiber transcriptome. PLoS genetics 10 (1):e1004073. doi:10.1371/journal.pgen.1004073

Yu Z, Haberer G, Matthes M, Rattei T, Mayer KF, Gierl A, Torres-Ruiz RA (2010) Impact of natural genetic variation on the transcriptome of autotetraploid Arabidopsis thaliana. Proc Natl Acad Sci U S A 107 (41):17809-17814. doi:10.1073/pnas.1000852107

Zentner GE, Henikoff S (2012) Surveying the epigenomic landscape, one base at a time. Genome biology 13 (10):250-250

Zhang D, Pan Q, Tan C, Zhu B, Ge X, Shao Y, Li Z (2016) Genome-wide gene expressions respond differently to A-subgenome origins in Brassica napus synthetic hybrids and natural allotetraploid. Front Plant Sci 7:1508. doi:10.3389/fpls.2016.01508

Zhang T, Hu Y, Jiang W, Fang L, Guan X, Chen J, Zhang J, Saski CA, Scheffler BE, Stelly DM, Hulse-Kemp AM, Wan Q, Liu B, Liu C, Wang S, Pan M, Wang Y, Wang D, Ye W, Chang L, Zhang W, Song Q, Kirkbride RC, Chen X, Dennis E, Llewellyn DJ, Peterson DG, Thaxton P, Jones DC, Wang Q, Xu X, Zhang H, Wu H, Zhou L, Mei G, Chen S, Tian Y, Xiang D, Li X, Ding J, Zuo Q, Tao L, Liu Y, Li J, Lin Y, Hui Y, Cao Z, Cai C, Zhu X, Jiang Z, Zhou B, Guo W, Li R, Chen ZJ (2015) Sequencing of allotetraploid cotton (Gossypium hirsutum L. acc. TM-1) provides a resource for fiber improvement. Nature biotechnology 33 (5):531-537. doi:10.1038/nbt.3207

Zhang W, Fan X, Gao Y, Liu L, Sun L, Su Q, Han J, Zhang N, Cui F, Ji J, Tong Y, Li J (2017) Chromatin modification contributes to the expression divergence of three TaGS2 homoeologs in hexaploid wheat. Scientific reports 7:44677. doi:10.1038/srep44677

Zhao N, Zhu B, Li M, Wang L, Xu L, Zhang H, Zheng S, Qi B, Han F, Liu B (2011) Extensive and heritable epigenetic remodeling and genetic stability accompany allohexaploidization of wheat. Genetics 188 (3):499-510. doi:10.1534/genetics.111.127688 